\title{
ICD-11 complex PTSD among Israeli male perpetrators of intimate partner violence: Construct validity and risk factors
}

\author{
Ohad Gilbar $^{\mathrm{a}, *}$, Philip Hyland ${ }^{\mathrm{b}, \mathrm{c}}$, Marylene Cloitre ${ }^{\mathrm{d}}$, Rachel Dekel ${ }^{\mathrm{a}}$ \\ a The Louis and Gabi Weisfeld School of Social Work, Bar-Ilan University, Ramat-Gan, Israel \\ b National College of Ireland, Dublin, Ireland \\ ${ }^{\mathrm{c}}$ Centre for Global Health, Trinity College Dublin, Dublin, Ireland \\ d National Center for PTSD Dissemination and Training Division, VA Palo Alto Healthcare System, Department of Psychiatry and Behavioral Science, Stanford University, \\ Palo Alto, CA, United States
}

\section{A R T I C L E I N F O}

\section{Keyword:}

PTSD

CPTSD

ICD-11

International trauma questionnaire (ITQ)

Intimate partner violence

Confirmatory factor analysis (CFA)

\begin{abstract}
A B S T R A C T
The International Classification of Diseases 11th Version (ICD-11) will include Complex Posttraumatic Stress Disorder (CPTSD) as a unique diagnostic entity comprising core PTSD and DSO (disturbances in self-organization) symptoms. The current study had three aims: (1) assessing the validity of CPTSD in a unique population of male perpetrators of intimate partner violence; (2) examining whether exposure to different types of traumatic events would be associated with the two proposed CPTSD factors, namely PTSD or DSO; and (3) assessing the differential association of various sociodemographic and symptom characteristics with each factor. Participants were 234 males drawn randomly from a sample of 2600 men receiving treatment at 66 domestic violence centers in Israel. Data were collected using the International Trauma Questionnaire (ITQ) - Hebrew version. Confirmatory factor analysis supported the factorial validity of ICD-11 CPTSD. Cumulative lifetime trauma and physical childhood neglect were associated with PTSD and DSO, while cumulative childhood violence exposure was associated only with DSO. Anxiety was associated only with DSO; depression more strongly with DSO than PTSD. Religious level contributed only to PTSD; compulsory military service only to DSO. The study supports the distinction between PTSD and DSO in the CPTSD construct and introduces the role of cultural variables.
\end{abstract}

\section{Introduction}

Two sibling trauma-based disorders have been proposed for the International Classification of Diseases 11th Version (ICD-11), to be published by the World Health Organization (WHO) in 2018 (Hyland, Shevlin, Elklit, et al., 2016). The first, Posttraumatic Stress Disorder (PTSD), is characterized by three main groups of symptoms: re-experiencing (Re), avoidance (Av), and sense of threat (Th) (Brewin et al., 2017). The WHO ICD-11 Working Group has recommended a refocus on the diagnosis of PTSD on the basis of three core elements, and the removal of non-specific symptoms that are also part of other disorders (Maercker et al., 2013), a formulation which has been supported by several factor structure analyses (Brewin et al., 2017). The second, Complex PTSD (CPTSD), describes more pervasive psychological distress that typically occurs following traumatic exposure of a chronic nature. In addition to the core symptom clusters of PTSD, the ICD-11 CPTSD definition includes three areas of disturbances in self-organization (DSO): affective dysregulation (AD), negative self-concept (NSC), and disturbances in relationships (DR) (Brewin et al., 2017). Several studies have indicated support for the construct validity of these two diagnoses in a variety of samples (Cloitre, Garvert, Brewin, Bryant, \& Maercker, 2013; Elklit, Hyland, \& Shevlin, 2014; Hyland, Shevlin, Elklit, et al., 2016; Karatzias et al., 2016; Perkonigg et al., 2015). However, not all have shown results supporting this conceptual model (Wolf et al., 2014); thus, the need for additional testing. Moreover, the model should be tested among new community and clinical samples from a variety of cultural contexts. Of particular interest are mandated patients. These individuals may have significant levels of posttraumatic stress symptoms (Hoyt, Wray, Wiggins, Gerstle, \& Maclean, 2012), as well as disturbances in self-organization, given their history of chronic exposure to traumatic events in childhood. However, because they tend not to seek help for traumatic distress, they are rarely studied in relation to CPTSD symptoms. Therefore, the purpose of this study was to evaluate the construct and discriminative validity of CPTSD in a sample of male perpetrators of intimate partner violence mandated for treatment.

In this study, confirmatory factor analysis (CFA) was used, an approach which has been used previously to test the validity of the proposed model (Hyland, Shevlin, Elklit, et al., 2016; Knefel \& LuegerSchuster, 2013; Nickerson et al., 2016; Shevlin et al., 2017) in other

\footnotetext{
* Corresponding author.

E-mail addresses: ohad.gilbar@biu.ac.il (O. Gilbar), Philip.hyland@ncirl.ie (P. Hyland), Marylene.cloitre@va.gov (M. Cloitre), Rachel.Dekel@biu.ac.il (R. Dekel).
} 
samples. The International Trauma Questionnaire (ITQ) was developed explicitly to capture the ICD-11 PTSD and CPTSD diagnoses, via selfreport measures (ITQ; Cloitre, Roberts, Bisson, \& Brewin, in preparation). To date, only three studies testing the content and construct validity of the ICD-11 diagnoses of PTSD and CPTSD with the ITQ have been published (Hyland, Shevlin, Brewin, et al., 2017; Karatzias et al., 2017; Murphy, Elklit, Dokkedahl, \& Shevlin, 2016). There is thus a need to further test the construct validity of ICD-11 PTSD and CPTSD with the ITQ in additional samples.

The differential diagnosis between ICD-11 PTSD and CPTSD is determined by the symptom profile rather than the individual's trauma history (Maercker et al., 2013). However, consistent with previous empirical investigations and conceptual models of complex PTSD (Ford \& Courtois, 2014; Herman, 1992; Van der Kolk, 1987), the types of events associated with the risk for ICD-11 CPTSD are expected to be sustained, repeated, or multiple forms of exposure (e.g., childhood abuse, domestic violence) and are of an interpersonal nature from which escape is difficult or impossible.

Findings related to the role of trauma history variables (e.g., type of trauma, its chronicity, and number of exposures to traumatic events) as risk factors for CPTSD have been somewhat mixed. Many studies have found a positive association between chronic childhood trauma exposure and a CPTSD symptom profile (Cloitre et al., 2013; Dokkedahl, Oboke, Ovuga, \& Elklit, 2015; Knefel \& Lueger-Schuster, 2013). The experience of cumulative childhood exposure to various types of interpersonal trauma (Hyland, Murphy, Shevlin, et al., 2017; Karatzias et al., 2017) has been more strongly associated with CPTSD than with PTSD symptom profiles. However, one study found no relationship between childhood abuse and CPTSD (Wolf et al., 2014), and another found no relationship between other types of childhood trauma and CPTSD (Krammer, Kleim, SimmenJanevska, \& Maercker, 2015). Additionally, only two studies have examined this subject by comparing PTSD and DSO cluster symptoms as delineated by the CPTSD definition. Endorsement of childhood abuse of any type (sexual, physical, emotional) and childhood physical or emotional neglect was more strongly associated with DSO than with PTSD symptom profiles (Shevlin et al., 2017); one study among childhood sexual abuse survivors, however, found that frequency of abuse was more strongly associated with PTSD than with DSO symptom profiles (Hyland, Shevlin, Elklit, et al., 2016). Given the conflicting evidence, and the scant research that has been conducted assessing trauma history characteristics that may distinguish PTSD and DSO cluster symptoms, it is important to further study predictors and correlates that might enable a differentiation between the two.

Several studies have found that, overall, ICD-11 CPTSD is associated with a greater number of co-morbid disorders (Perkonigg et al., 2015) and greater severity of comorbid symptoms (Elklit et al., 2014; Murphy et al., 2016) than is ICD-11 PTSD. One study looked at specific differential associations of comorbid symptoms in relation to the two CPTSD factors (Hyland, Shevlin, Elklit, et al., 2016). Findings indicated that a higher level of anxiety was more strongly associated with PTSD symptoms than DSO symptoms, while a higher level of dysphoria was associated with DSO. These results are consistent with the conceptualization of PTSD symptoms arising from the experience of threat, while those of DSO are typically associated with the loss of psychological (e.g., self-efficacy) and environmental (e.g., social networks) resources that can result from repeated or sustained traumatic events.

Additional possible risk factors for CPTSD are sociodemographic. The CPTSD profile has been associated with lower educational attainment and lower socioeconomic status (Perkonigg et al., 2015), unemployment (Hyland, Murphy, Shevlin, et al., 2017), and a lower likelihood of full-time or part-time employment (Karatzias et al., 2017). It remains to be seen whether such differences will be observed in the Israeli patient population.

Research supporting the validity of the ICD-11 CPTSD construct has, to date, been conducted among quite varied clinical and community samples who have been exposed to interpersonal violence. These include individuals who experienced both adult and childhood interpersonal violence (Cloitre et al., 2013; Karatzias et al., 2017), childhood sexual abuse victims (Hyland, Shevlin, Elklit, et al., 2016; Hyland, Shevlin, McNally, et al., 2016), survivors of institutional abuse (Knefel, Garvert, Cloitre, \& Lueger-Schuster, 2015), a community sample of young adults exposed to interpersonal violence (Perkonigg et al., 2015), and a non-western (Ugandan) young adult sample exposed to civil war, including those abducted as children for soldiering (Dokkedahl et al., 2015; Murphy et al., 2016).

No studies have specifically focused on the traumatic experiences of men and, more particularly, men who have been identified as perpetrators of violence. Studies indicate that these men have usually experienced traumatic events at a greater frequency than other men in the community (Maguire et al., 2015), specifically exposure to violence by their parents (Delsol \& Margolin, 2004). They also experience PTSD at higher rates than do community samples (Delsol \& Margolin, 2004; Hoyt et al., 2012). Nevertheless, they have rarely been the subjects of studies in this area because they generally tend not to seek help for their traumatic distress. Consequently, they comprise a new and relevant clinical population by which to validate the ICD-11 PTSD definitions.

The first aim of the study was to test the latent structure of CPTSD symptoms via the Hebrew version of the ITQ among a special study sample of males who perpetrated intimate partner violence (IPV) and are being treated by social service agencies. The second was to examine the association between types of traumatic events and the PTSD versus DSO symptom clusters. The current study specifically examined the potential differential contributions of exposure to child abuse, physical neglect, and cumulative traumas to the risk for PTSD versus DSO, as well as the relative contribution of anxiety and depressive symptomatology. Finally, while the validation of this structural definition has been tested in several cultural contexts such as America (Cloitre et al., 2013), Denmark (Hyland, Shevlin, McNally, et al., 2016), Austria (Knefel et al., 2015), Germany (Perkonigg et al., 2015), Britain (Hyland, Shevlin, Brewin, et al., 2017; Karatzias et al., 2017), Uganda (Murphy, Elklit, Dokkedahl, \& Shevlin, 2016) and Papua New Guinea (Tay, Rees, Chen, Kareth, \& Silove, 2015; Silove, Tay, Kareth, \& Rees, 2017), it is critical that investigations of the ICD-11 PTSD definitions continue to be tested in other cultural contexts (Hyland, Shevlin, Elklit, et al., 2016). In this study, therefore, we looked at variables that represent the Israeli cultural context, including level of religiousness and time in compulsory military service. In a previous study, both time in compulsory military service and religiousness were connected to PTSD. Time in service may be viewed as a risk factor as it is related to increased exposure to combat and other traumatic events (Solomon \& Horesh, 2007). Religiosity may contribute to PTSD due to inner turmoil arising from potential conflict generated between religious principles and behavior, in addition to the already distressing fear and horror of the event (Ankri, Bachar, \& Shalev, 2010). The following hypotheses were formed. First, measurement models of CPTSD that distinguish between PTSD and DSO symptoms clusters will exhibit a superior model fit. Second, the Hebrew version of the ITQ will possess satisfactory internal reliability. Third, greater exposure to traumatic events and/or child abuse and physical neglect will be a stronger predictor of the DSO than the PTSD symptom cluster. Fourth, the DSO symptom cluster will be predicted by greater severity of depression than will the PTSD symptom cluster, and the PTSD symptom cluster will be predicted by greater severity of anxiety than will the DSO. Finally, socio-demographic variables such as low level of education, income, and employment will predict the DSO symptom cluster more often than the PTSD symptom cluster. The specific cultural variable of religious level will predict PTSD more often than DSO, as religious beliefs can be shattered by traumatic events: i.e., that which once served as a source of strength may become a stressor and lead to the experience of PTSD symptoms. In addition, we hypothesize that time in the army will predict PTSD more often than DSO as time in the army brings greater exposure to combat experiences. 


\section{Methods}

\subsection{Participants and procedures}

This study was conducted in collaboration with Israel's Ministry of Social Welfare and Social Services, and ethical approval was received from both Bar-Ilan University's institutional review board and the Welfare Ministry's research department. Data collection took place from February-August 2016. Despite concerns raised in the literature regarding this population's cooperation with research studies, there was a response rate of approximately $70 \%$ in the current study.

Participants were 234 randomly-drawn males from the Jewish population in Israel who received treatment at 30 domestic violence prevention centers. They comprise about $9 \%$ of the total number of 2600 males who were treated at 66 centers during the same time period (Hasherut Lerevahat Haprat VeHamishpaha, 2016). The majority of respondents (88\%) sought help after a legal or social service intervention. Length of time in intervention ranged from two weeks to three years. The sociodemographic variables revealed that more than half $(56 \%)$ were in a relationship, $37.6 \%$ were separated, and $6.4 \%$ were single. Average length of education was 12.8 years $(\mathrm{SD}=2.58$; range $=8-21$ ). Regarding religiosity, $37.2 \%$ were traditional (observing some Jewish rituals but not seeing themselves as religious), $47 \%$ secular, $11.2 \%$ religious, and $4.7 \%$ Ultraorthodox. In addition, $14.7 \%$ were unemployed, $9 \%$ had part-time jobs, and $76 \%$ had full-time jobs. Regarding compulsory military service, $65.5 \%$ fulfilled their whole period of service, $20.1 \%$ didn't serve at all, and $14.5 \%$ fulfilled partial service. The mean number of direct exposures to traumatic events was 4.23 (possible range 15, actual range 14; $S D=3.06$, $\mathrm{MD}=3.77$ ), the mean number of exposures to child abuse was 2.03 (possible range 6, actual range $6 ; S D=1.86$ ), and the mean number of exposures to childhood physical neglect was 1.29 (possible range 6, actual range 6; $S D=1.78$ ).

\subsection{Measures}

\subsubsection{CPTSD and PTSD: International Trauma Questionnaire (ITQ;} version 1.5) (Cloitre, roberts, bisson, \& brewin, unpublished measure)

The ITQ is a 23-item self-report measure - currently under development - for screening ICD-11 PTSD and CPTSD symptomatology. In its current preliminary form, the measure demonstrates good construct, factor, and discriminant validity (Hyland, Shevlin, McNally et al., 2016; Hyland, Shevlin, Brewin et al., 2017; Karatzias et al., 2016; Karatzias et al., 2017: Shevlin et al., 2017). Seven items represent the three clusters of PTSD (Re, Av, Th). There are three Re items (one is a test item and is not used for analytical purposes), two Av items, and two Th items. Symptom endorsement is scored on a Likert-type scale, indicating how bothersome a symptom has been over the past month with scores ranging from 0 (not at all) to 4 (extremely). Sixteen items represent the three DSO clusters (AD 9 items; NSC 4 items; DR 3 items), where endorsement of items indicates how typical the problem is to the individual, with scores ranging from 0 (not at all) to 4 (extremely). The scale can be used to estimate a self-reported ICD-11 PTSD or CPTSD diagnosis by re-coding the Likert scores into six binary variables. These represent each of the three PTSD and DSO symptom clusters based on the following cut-off scores. A diagnosis of PTSD requires a score of $>2$ (moderately) for at least one symptom in each of its three clusters. A diagnosis of CPTSD requires PTSD and the additional following scores for each of the three DSO clusters. The proposed algorithm for each DSO cluster requires a sum that is half of the total possible score of each of the scales: $\mathrm{AD}$ score $>10$ on items 1-5 (hyperactivation) or a score of $>8$ on items 6-9 (deactivation); NSC score $>8$ on items 10-13; and DR score of $>6$ on items 14-16. The diagnosis of CPTSD, like that for PTSD, requires functional impairment. Functional impairment is defined as a score $>2$ on a Likert-type scale indicating impairment due to symptoms ranging from 0 (not at all) to 4 (extremely). The current algorithm requires that impairment be evaluated and associated with both the PTSD and DSO symptom clusters.

Cronbach's alpha reliability estimates for the PTSD indicators in the current sample were acceptable $(\operatorname{Re}=0.72, \mathrm{Av}=0.82$, and $\mathrm{Th}=0.54)$ for the PTSD cluster $=0.75$, but higher for the DSO indicators $(\mathrm{AD}=0.84$, NSC $=0.84$, and $\mathrm{DR}=0.82)$, and for $\mathrm{DSO}=0.91$. The estimates of reliability for the indicators are likely to be underestimates of true reliability due to the small number of variables (Eisinga, Grotenhuis, \& Pelzer, 2012). In this study, this measure was translated into Hebrew according to the WHO criteria, which include reverse-translation.

\subsubsection{Exposure to traumatic events: life events checklist (LEC-5) (Weathers} et al., 2013)

The LEC-5 is a 17-item self-report measure designed to screen for potentially traumatic events (PTEs) in a respondent's lifetime. The LEC was originally used to assess criteria A in "The Clinician-Administered PTSD Scale" (CAPS) (Blake et al., 1990). The validation of the LEC has been supported by the exhibit of adequate temporal stability, and shows good convergence with an established measure of trauma history (Gray, Litz, Hsu, \& Lombardo, 2005). This measure assesses lifetime exposure to 15 traumatic events (e.g., natural disaster, sexual assault, lifethreatening illness/injury), plus two more - "any other very stressful event/experience" and "serious injury, harm, or death you caused to someone else" - which can be used to indicate exposure to a trauma that was not listed. For each item, the respondent checks, in regard to the event: (1) "happened to me," (2) "witnessed it happening to somebody else," (3) "learned about it happening to someone close to me," (4) "was part of my job," (5) "not sure it applies," or (6)" doesn't apply to my experience." In order to create a summed total that represents the number of different life events that the respondent has experienced, the items were re-coded into binary variables with "happened to me" responses being coded as 1 and all other responses coded as 0 . This coding produced a single total cumulative index variable with possible scores ranging from 0 to 17 .

2.2.3. Exposure to violence and physical neglect in childhood: conflict tactics scale (CTS) (Straus, hamby, boney-McCoy, \& sugarman, 1996)

Based on a previous study (Lee, Walters, Hall, \& Basile, 2013), individuals exposed to violence in childhood were distinguished from individuals exposed to childhood physical neglect according to this measure, adapted from the validated version of Straus et al. (1996). The measure was constructed from items which asked respondents to rate the frequency with which their father or male guardian or mother or female guardian used specific conflict tactics against (a) each other, and (b) the respondent. Exposure to violence in childhood was assessed in accordance with conflict tactics, described as follows: (1) insulted, yelled at, sworn at, or threatened to be hit or have something thrown at; (2) threw something, pushed, grabbed, shoved, or slapped; (3) kicked, hit with a fist, hit with something; or (4) beat up, or threatened with a knife or gun. These tactics reflect the experiences of psychological, physical, and severe physical abuse. Cronbach's alpha $=0.85$. The scores for these items ranged from 0 to 36. Physical neglect in childhood was measured by asking a two-part question: "By the time you started 12th grade, how often had your parents or other adult caregivers (a) not taken care of your basic needs, such as keeping you clean or providing you with food or clothing, and (b) left you home alone when an adult should have been with you?" Responses for each of the two parts of the question ranged from $0=$ never to $6=$ more than 20 times. The scores for these items ranged from 0 to 12 . Cronbach's alpha for the two items of physical neglect was $=0.54$.

\subsubsection{Depression and anxiety: brief symptom inventory (BSI) (Derogatis \& melisaratos, 1983)}

Participants' depression and anxiety levels were measured using the appropriate subscales of the Brief Symptoms Inventory. The BSI showed good convergence with a similar measure of anxiety and depression and was validated by construct validity (BSI; Derogatis \& Melisaratos, 

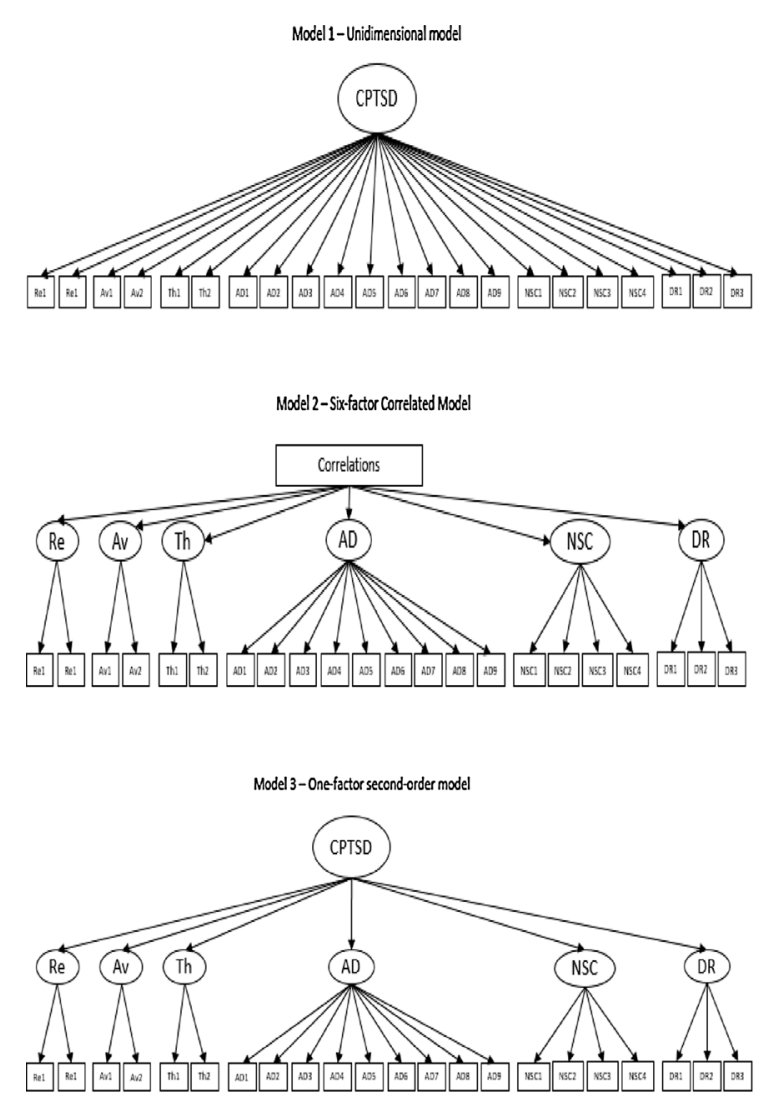
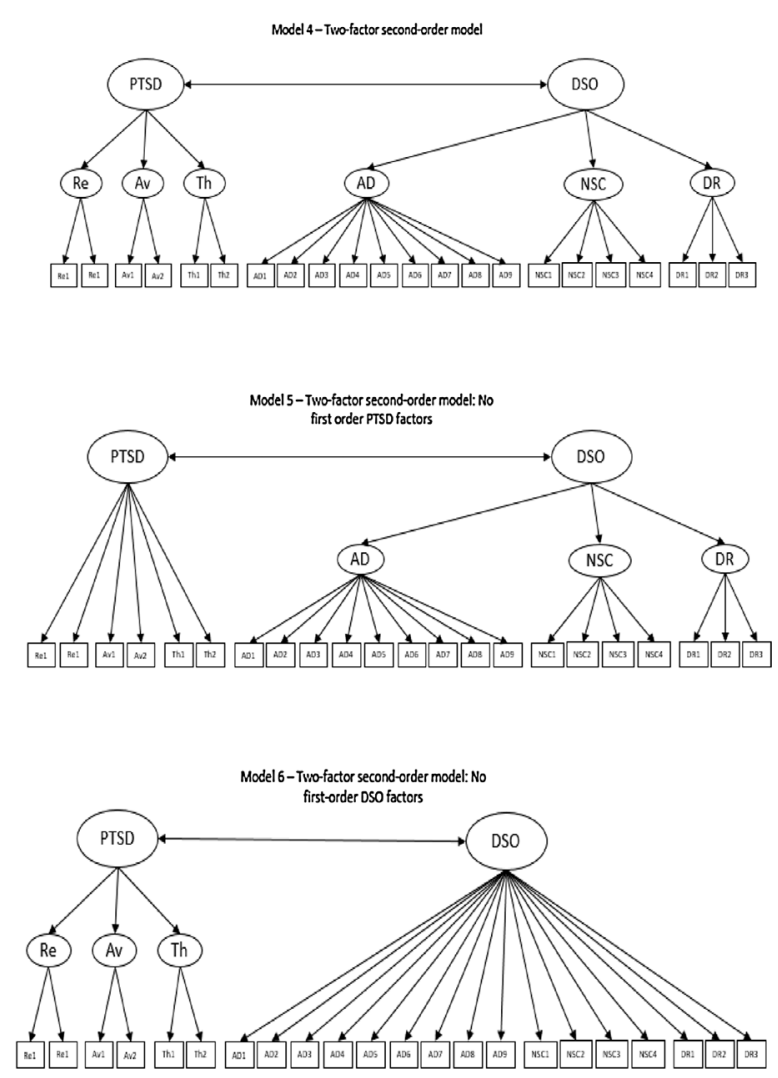

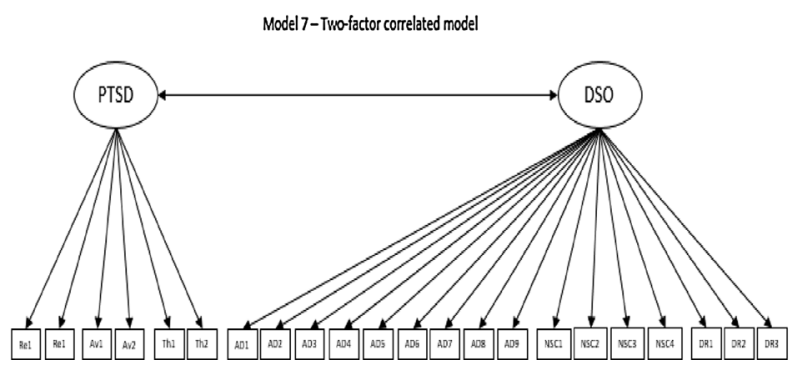

Fig. 1. Alternative models of the latent structure of Complex PTSD symptoms.

1983). Participants were asked to rank the degree to which they experienced each depressive and anxiety symptom during the previous month on a scale ranging from 1 (not at all) to 4 (very much). We used the Hebrew translation (Gilbar \& Ben-Zur, 2002). Cronbach's alpha for depression was 0.83 and for anxiety was 0.83 .

\subsubsection{Background variables}

Education, income, religious level, time in the army's compulsory military service, and employment were assessed

\subsection{Statistical analysis}

The latent structure of the ITQ was tested using confirmatory factor analysis (CFA) based on responses to the full pool of 22 items. Seven alternative models were specified (see Fig. 1) and tested as representative of the PTSD and DSO symptom clusters of CPTSD. Overall, the aim of testing alternative models was to determine whether: (1) the PTSD and DSO symptom clusters were distinct dimensions, (2) the PTSD symptom cluster was better represented as three correlated dimensions rather than one dimension, (3) DSO was better represented as three correlated dimensions rather than one dimension, and (4) there was a hierarchical structure (second-order factors) that explained the associations between the first-order PTSD and DSO dimensions. Model 1 is a one-factor model where all symptoms load on the single latent variable CPTSD. Model 2 is a correlated six-factor model (Re, Av, Th, $\mathrm{AD}$, NSC, and DR). Model 3 explains the factor correlations in Model 2 with a single second-order factor representing CPTSD. Model 4 (reflecting the ICD-11 proposals; Maercker et al., 2013) specifies two correlated second-order factors (PTSD and DSO) to explain the covariation among the six first-order factors; Re, Av, and Th load on the PTSD factor, and AD, NSC, and DR load on the DSO factor. Model 5 tests the hypothesis that there is no hierarchical structure for the PTSD items, but there is a hierarchical structure for the DSO items; and Model 6 tests the hypothesis that there is no hierarchical structure for the DSO items, but there is a hierarchical structure for the PTSD items. Model 7 proposes that all of the PTSD and DSO items load on two correlated firstorder factors. For all models, the error variances were specified as uncorrelated. Each model was specified and estimated by Mplus 7 (Muthén \& Muthén, 2013) using the mean- and variance-adjusted weighted least squares estimator (WLSMV) based on the polychromic correlation matrix of latent continuous response variables. The WLSMV estimator is the most appropriate statistical treatment of ordinal 
indicators in a CFA context (Brown, 2006). Variables were treated as categorical variables for the purpose of a polychromic matrix. Other methods of analysis, such as maximum likelihood estimation, tend to produce incorrect standard errors, attenuate the relationships between observed variables, and produce possible pseudo-factors (Brown, 2006). The WLSMV estimator has been shown to produce correct parameter estimates, standard errors, and test statistics (Flora \& Curran, 2004).

The amount of missing data for the ITQ was low. It did not exceed $4 \%$, and the minimum covariance coverage was $92 \%$. This was handled using pairwise present analysis, which is the default when the WLSMV estimator is used (Asparouhov \& Muthén, 2010). Goodness of fit for each model was assessed with a range of fit indices including the chisquare, the comparative fit index (CFI), and the Tucker-Lewis Index (TLI). A non-significant $x^{2}$ and values greater than 0.90 for the CFI and TLI are considered to reflect acceptable model fit. Additionally, the Root Mean Square Error of Approximation (RMSEA) was reported, with a value less than 0.05 indicating close fit and values up to 0.08 indicating reasonable errors of approximation (Hu \& Bentler, 1999). When the best model was identified, factor scores were calculated. MLR estimation was used simply to generate the BIC values so that alternative models could be statistically compared.

Following the identification of the best-fitting model of ICD-11 CPTSD, a range of predictors was added to the model to assess their differential predictive effects on the identified latent variables. Ten predictors were added to the CFA model. Three related to trauma history: number of exposures to traumatic events, frequency of acts of physical abuse in childhood, and frequency of acts of physical neglect experienced in childhood. Two were related to current symptoms: BSI scores of anxiety and depression. Five were related to sociodemographic variables: education, income, religiosity, time in the army, and employment. Testing differential predictive effects proceeded in a sequential fashion. The predictors were first entered into the model with the best CFA solution, and each factor was regressed onto each predictor with the regression. Composite reliability for the preferred model was also calculated. Composite reliability estimates the internal consistency of a set of items without the strict assumptions of tauequivalence (Raykov, 1997) and allows for the reliability of a smaller set of variables to be estimated than is possible with Cronbach's alpha.

\section{Results}

See Table 1 for frequency of symptom endorsement for each PTSD and DSO item of the ITQ, based on diagnostic estimates. The average of the PTSD symptoms was 0.80 ( $\mathrm{SD}=0.73$, Range $=0-3$ ), and the average of the DSO symptoms was 0.97 ( $\mathrm{SD}=0.97$, Range $=0-4$ ). Among the current sample of male IPV perpetrators, following the ICD11 binary diagnostic categorization into either PTSD (PTSD cluster alone) or CPTSD (PTSD plus DSO clusters), 8.1\% met the criteria for PTSD $(n=18)$, and $1.4 \%(n=3)$ met the criteria for CPTSD.

To reduce confirmatory bias, we only included in the confirmatory model respondents who provided responses to more than 50 percent of the items in the PTSD and the DSO scales, which means that in this study, the participant number was reduced by 11 participants $(\mathrm{N}=223)$. The fit indices for the alternative CPTSD models are presented in Table 1. Models $1,5,6$, and 7 were rejected as poor approximations of the data. Models 2, 3 , and 4 were all found to offer a reasonable fit to the data. Chi-square difference testing using the DIFFTEST option showed no statistically significant differences between Models 2, 3, and 4. However, the BIC value was lowest for Model 4, suggesting that this model was statistically superior. In addition, as Model 4 reflects the proposed ICD-11 models of CPTSD, this model was favored on the grounds of statistical superiority and theoretical consistency. Factor loadings for the higher-order model are reported in Table 2.

Factor loading for the higher order model are reported in Table 3. All symptom indicators loaded onto their respective first-order latent factors in an expected manner with the loadings being positive, high $(>.45$ in all cases), and statistically significant $(\mathrm{p}<.001)$. Moreover,
Table 1

ITQ CPTSD symptom frequency organized by PTSD and DSO factors, based on diagnostic estimates.

\begin{tabular}{ll}
\hline & Valid\% (n) \\
\hline PTSD Factor & \\
Upsetting dreams (Re1) & $9.8(22)$ \\
Reliving the event in the here and now (Re2) & $14(31)$ \\
Internal avoidance (Av1) & $28.7(64)$ \\
External avoidance (Av2) & $19.8(42)$ \\
Being on guard (Th1) & $46.1(101)$ \\
Jumpy/startled (Th2) & $19.8(42)$ \\
DSO Factor & \\
Intense reactions (AD1) & $30.5(68)$ \\
Long time to calm down (AD2) & $42.1(94)$ \\
Feelings easily hurt (AD3) & $53(116)$ \\
Uncontrollable anger (AD4) & $31.4(70)$ \\
Reckless behavior (AD5) & $32.3(72)$ \\
Numb (AD6) & $30.4(68)$ \\
Difficulty feeling pleasure (AD7) & $25.7(57)$ \\
World is distant (AD8) & $23.2(52)$ \\
Feeling outside of body (AD9) & $10.7(24)$ \\
Failure (NSC1) & $23.2(51)$ \\
Worthless (NSC2) & $17.9(39)$ \\
Self-shame (NSC3) & $24.9(55)$ \\
Guilt (NSC4) & $48.7(108)$ \\
Cut-off from others (DR1) & $21.3(47)$ \\
Difficult to stay close to others (DR2) & $25.1(55)$ \\
Avoiding relationships (DR3) & $20.3(44)$ \\
\hline
\end{tabular}

Note: $\quad$ PTSD = Posttraumatic Stress Disorder diagnostic; Re = Re-Experiencing; Av = Avoidance; $\quad$ Th $=$ Sense of Current Threat; DSO $=$ Disturbances in SelfOrganization; $\quad \mathrm{AD}=$ Affective $\quad$ Dysregulation; $\quad \mathrm{NSC}=$ Negative Self Concept; $\mathrm{DR}=$ Disturbances in Relationships.

the Re, Av, and Th factors all loaded strongly onto the PTSD higherorder factor. Likewise, the AD, NSC, and DR factors loaded strongly onto the DSO higher-order factor. Composite reliability findings, based on estimates derived from the CFA analysis, indicated that the ICD-ITQ possesses excellent internal reliability. The six first-order subscales demonstrated high levels of reliability $(\mathrm{Re}=0.82, \mathrm{Av}=0.90$, $\mathrm{Th}=0.66, \mathrm{AD}=0.88, \mathrm{NSC}=0.90$, and $\mathrm{DR}=0.87$ ), as did the six PTSD (.92) and 16 DSO (.95) items.

Following the selection of the higher-order model structure of the CPTSD symptoms, ten predictors were added to the model. The CFI, TLI, and RMSEA values for the models in this step were indicative of adequate model fit: CFI $=0.940$, TLI $=0.932$, RMSEA $=0.053$ [0.045 0.061] . Table 4 reports the path coefficients for the ten predictors of the PTSD and DSO factors in detail. Income, education, and employment were not related to PTSD or DSO. Religiosity was a predictor of PTSD $(\beta=0.22$, $\mathrm{p}<.05$ ) but was not related to DSO symptoms. The opposite effect was observed for time in the army: it was a negative predictor of DSO $(\beta=-0.24, p<.01)$ and was not related to PTSD symptoms. Number of traumatic life events across the entire lifespan and childhood physical neglect were associated with both PTSD symptoms $(\beta=0.32, \mathrm{p}<.001$ and $\beta=0.26, p<.001$, respectively) and with DSO symptoms ( $\beta=0.24, p<.01$ and $\beta=0.13, p<.01$, respectively). However, cumulative exposure to violence in childhood (witnessing or experiencing verbal or physical violence in the home) was associated with DSO symptoms $(\beta=0.09, \mathrm{p}<.05)$ but not with PTSD symptoms.

In addition, an increased level of anxiety was a predictor of DSO symptoms $(\beta=0.16, p<.05)$ and was not related to PTSD symptoms. An increased level of depression was a predictor of both PTSD symptoms $(\beta=0.27, p<.05)$ and DSO symptoms $(\beta=0.53, p<.001)$. In this step we also added the correlations between anxiety and depression ( $\beta=0.43, p<.001)$ and cumulative childhood exposure to violence and physical neglect (not significant).

These results indicate that religiosity was associated with PTSD. Cumulative lifetime trauma and childhood physical neglect were associated with both PTSD and DSO symptom clusters, while cumulative exposure to childhood violence was associated only with DSO. 
Table 2

Model fit statistics for the alternative models of the ICD-TQ.

\begin{tabular}{|c|c|c|c|c|c|c|c|c|}
\hline Models & $\mathrm{X}^{2}$ & $\mathrm{df}$ & CFI & TLI & RMSEA [90\%CI] & SRMR & AIC & BIC \\
\hline Unidimensional model & $927.68^{*}$ & 209 & 0.829 & 0.811 & $0.124(0.116,0.132)$ & 0.091 & 13002.463 & 13227.336 \\
\hline Two-factor model & $514.18^{*}$ & 194 & 0.924 & 0.909 & $0.086(0.077,0.095)$ & 0.077 & 12649.163 & 12925.143 \\
\hline Correlated six-factor & $534.46^{*}$ & 203 & 0.921 & 0.910 & $0.086(0.077,0.094)$ & 0.079 & 12659.038 & 12904.355 \\
\hline Higher-order model & $504.60^{*}$ & 202 & 0.928 & 0.917 & $0.082(0.073,0.091)$ & 0.078 & 12646.224 & 12894.947 \\
\hline Two-factor second order model (No PTSD) & $578.58^{*}$ & 205 & 0.911 & 0.900 & $0.900(0.082,0.099)$ & 0.089 & 12726.650 & 12965.152 \\
\hline Two-factor second order model (No DSO) & $668.31^{*}$ & 205 & 0.890 & 0.876 & $0.102(0.092,0.109)$ & 0.081 & 12819.455 & 13058.047 \\
\hline Two-factor correlated model (correlated model) & $736.42^{*}$ & 208 & 0.874 & 0.860 & $0.107(0.098,0.115)$ & 0.090 & 12899.822 & 13128.103 \\
\hline
\end{tabular}

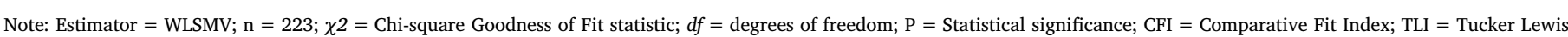

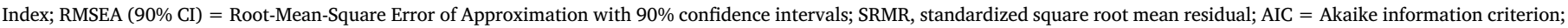
$\mathrm{BIC}=$ Bayesian Information Criterion; Best fitting model in bold. ${ }^{*} p<.001$.

Regarding symptoms, anxiety was associated only with DSO symptoms, and higher levels of dysthymia were more strongly associated with DSO symptoms than PTSD symptoms. Overall, the ten covariates explained $48 \%$ of the variance in PTSD symptomology and $68 \%$ of the variance in DSO symptomology.

\section{Discussion}

The primary aim of this study was to test the latent structure of the new ICD-11 CPTSD definitions via the Hebrew version of the ITQ among a sample of Israeli male IPV perpetrators. The CFA results strengthen the findings from two previous studies which used the same method (Hyland, Shevlin, Elklit, et al., 2016; Karatzias et al., 2016). The two-factor higherorder model of CPTSD had the best fit, compared to six other alternative models. The fit of this model to the data was considered acceptable, based on established criteria for assessment of model fit (Kline, 2011). In this study, the closest alternative model was the two-factor model, a finding which contrasts with previous research showing very similar fit statistics produced by the correlated six-factor model. However, based on the lower BIC, the six-factor model has at least an equivalent fit to the two-factor model, something which was also shown in previous research (Hyland, Shevlin, Elklit, et al., 2016). Therefore, although the higher-order CPTSD model was deemed to provide the best fit based on grounds of parsimony (degree of freedom) and theoretical consistency, it is important to test this alternative model in further research. In addition, the ITQ was found to possess adequate composite reliability.

This study was performed with a unique clinical population for the first time: male patients mandated as a result of IPV. The current findings identified a high prevalence of traumatic events and high rates of PTSD symptoms consistent with findings of other studies (Maguire et al., 2015; Taft, Murphy, \& Creech, 2016; Taft, Schumm, Marshall, Panuzio, \& Holtzworth-Munroe, 2008), but in addition identified the presence of DSO symptoms which were systematically related to PTSD symptoms as predicted by the CPTSD model.

The low CPTSD diagnosis rate was surprising, especially compared to the rates of PTSD based on the dichotomous criteria of the ICD-11. The percent endorsement of items in the Affective Dysregulation construct was relatively high and consistent with previous research in this population (Maguire et al., 2015). The guilt item in the Negative SelfConcept construct was also high. Notable, however, was the relatively

Table 3

Standardized factor loadings (standard errors) for Model 4.

\begin{tabular}{|c|c|c|c|c|c|c|}
\hline Items & $\operatorname{Re}$ & $\mathrm{Av}$ & Th & $\mathrm{AD}$ & NSC & $\mathrm{DR}$ \\
\hline Upsetting dreams (Re1) & $.86(0.07)$ & & & & & \\
\hline Reliving the event in the here and now (Re2) & $.81(0.08)$ & & & & & \\
\hline Internal avoidance (Av1) & & $0.98(0.04)$ & & & & \\
\hline External avoidance (Av2) & & $0.83(0.04)$ & & & & \\
\hline Being on guard (Th1) & & & $0.62(0.06)$ & & & \\
\hline Jumpy/Startled (Th2) & & & $0.78(0.05)$ & & & \\
\hline Intense reactions ( $\mathrm{AD} 1)$ & & & & $0.68(0.04)$ & & \\
\hline Long time to calm down (AD2) & & & & $0.66(0.03)$ & & \\
\hline Feelings easily hurt (AD3) & & & & $0.66(0.04)$ & & \\
\hline Uncontrollable anger (AD4) & & & & $0.76(0.03)$ & & \\
\hline Reckless behavior (AD5) & & & & $0.45(0.06)$ & & \\
\hline Numb (AD6) & & & & $0.59(0.04)$ & & \\
\hline Difficulty feeling pleasure (AD7) & & & & $0.76(0.04)$ & & \\
\hline World is distant (AD8) & & & & $0.78(0.03)$ & & \\
\hline Feeling outside of body (AD9) & & & & $0.72(0.04)$ & & \\
\hline Failure (NSC1) & & & & & $0.89(0.02)$ & \\
\hline Worthless (NSC2) & & & & & $0.93(0.02)$ & \\
\hline Self-shame (NSC3) & & & & & $0.82(0.03)$ & \\
\hline Guilt (NSC4) & & & & & $0.69(0.04)$ & \\
\hline Cut-off from others (DR1) & & & & & & $0.91(0.02)$ \\
\hline Difficult to stay close to others (DR2) & & & & & & $0.83(0.03)$ \\
\hline Avoiding relationships (DR3) & & & & & & $0.76(0.04)$ \\
\hline Second-order factor loadings & & & PTSD & & DSO & \\
\hline Re-experiencing (Re) & & & $0.54(0.07)$ & & & \\
\hline Avoidance (Av) & & & $0.81(0.04)$ & & & \\
\hline Sense of current threat (Th) & & & $0.97(0.06)$ & & & \\
\hline Affective dysregulation (AD) & & & & & $0.90(0.02)$ & \\
\hline Negative self-concept (NSC) & & & & & $0.80(0.03)$ & \\
\hline Disturbances in relationships (DR) & & & & & $0.87(0.03)$ & \\
\hline
\end{tabular}

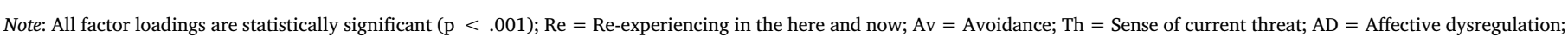
NSC $=$ Negative self-concept; DR $=$ Disturbed relationships. 
Table 4

Unstandardized (Standard Error) and Standardized Beta Values for the Predictors of Symptoms of PTSD and DSO from the Final Stage of Analysis.

\begin{tabular}{|c|c|c|c|c|c|c|}
\hline \multirow[t]{2}{*}{ Predictors variables } & \multicolumn{3}{|l|}{ PTSD } & \multicolumn{3}{|l|}{ DSO } \\
\hline & $\mathrm{B}(\mathrm{SE})$ & $\beta$ & $\mathrm{p}$ & $\mathrm{B}(\mathrm{SE})$ & $\beta$ & $\mathrm{P}$ \\
\hline Religious level & $0.122(0.056)$ & 0.221 & $<.05$ & $0.073(0.062)$ & 0.093 & .233 \\
\hline Time in army & $-0.106(0.057)$ & -0.184 & .061 & -0.197 (0.069) & 0.241 & $<.01$ \\
\hline Employment & $0.074(0.056)$ & 0.112 & .176 & $0.023(0.081)$ & 0.024 & .779 \\
\hline Income & $0.028(0.038)$ & -0.080 & .446 & $0.030(0.044)$ & 0.061 & .492 \\
\hline Education (years) & $0.016(0.017)$ & 0.093 & .321 & $0.022(0.021)$ & 0.088 & .306 \\
\hline Lifetime PTEs & $0.122(0.039)$ & 0.325 & $<.001$ & $0.132(0.042)$ & 0.249 & $<.01$ \\
\hline Anxiety & $0.127(0.071)$ & 0.202 & .053 & $0.146(0.066)$ & 0.164 & $<.05$ \\
\hline Depression & $0.159(0.064)$ & 0.271 & $<.05$ & $0.442(0.060)$ & 0.532 & $<.001$ \\
\hline Exposure to violence in Childhood & $0.007(0.019)$ & 0.027 & .706 & $0.035(0.018)$ & 0.095 & $<.05$ \\
\hline Childhood physical neglect & $0.076(0.023)$ & 0.262 & $<.001$ & $0.054(0.019)$ & 0.132 & $<.01$ \\
\hline$\%$ variance explained & & $50 \%$ & & & $65 \%$ & \\
\hline
\end{tabular}

Note. PTSD = posttraumatic stress disorder; DSO = disturbances in self-organization; PTEs = optional traumatic events SE $=$ standard error.

low endorsement of items related to Disturbances in Relationships, the third DSO symptom cluster. It may be that despite having been identified as perpetrating interpersonal violence and being mandated to treatment, the participants did not see themselves as having interpersonal problems, a factor which itself may contribute to problems in interpersonal violence, as has been suggested in the literature previously (Bell \& Naugle, 2008).

Another contributor to the low CPTSD rate may be that among those who endorsed DSO symptoms, there was a relatively lower endorsement of PTSD symptoms, particularly symptoms of re-experiencing. It might be that the experience of very early traumatic events results in few clear memories, which in turn results in less re-experiencing of the events (Briere, 2002). Therefore, it is crucial that the WHO ICD-11 Working Group take these findings into consideration when they make the final determination regarding which items will constitute the reexperiencing cluster, as well as which items will constitute the AD symptom cluster of the DSO factor.

The second aim of the study was to determine whether the predictors of PTSD and DSO are differed. Childhood physical neglect was found in previous research to be one of the main risk factors for PTSD among this population (Taft et al., 2008). Our results indicate that they contribute to both the PTSD and the DSO symptom factor of CPTSD. Total life cumulative trauma was associated with the PTSD cluster, but cumulative childhood violence, comprised of both experiencing and witnessing verbal abuse and physical abuse, was associated only with the DSO cluster. These findings are consistent with the theory that DSO symptoms are associated with chronic exposure to repeated or multiple types of traumatic events particularly in childhood. The fact that childhood physical neglect contributed to both PTSD and DSO is of interest and calls for further investigation.

In addition, we studied the relative association of anxiety and depression to the two CPTSD factors. Our findings indicated that, as expected, depression was more strongly related to DSO than to PTSD. The association of anxiety to the two factors was essentially equivalent. This latter finding is not supportive of our hypothesis, which was based on earlier findings (Hyland, Shevlin, McNally, et al., 2016) that anxiety would be more strongly associated with the PTSD factor. Although PTSD and DSO are commonly co-morbid with general anxiety (Karatzias et al., 2016), high levels of comorbidity between anxiety, PTSD, and DSO could have been expected. These results suggest a crossover between the theoretical directions of the psychological symptoms and the two factors, and indicate the need for further studies to examine the relationship between these risk factors and CPTSD factors.

In relation to socio-demographic factors, only the variables that represented the Israeli cultural context predicted either PTSD or DSO. Level of religiosity predicted PTSD, as has been found in previous research on Israeli Jewish society (Ankri et al., 2010). In addition, less time served in the army predicted more DSO symptoms. This finding likely results from the fact that the Israeli army is highly selective when it comes to recruiting for compulsory service and tends to release those with psychological problems. It may be that DSO symptoms were already present and evident prior to recruitment given that, at least in theory, the risk for DSO symptoms is strongly associated with childhood trauma, and the majority of this sample had experienced childhood trauma. Additionally, this study was conducted among men, a unique research population considering the fact that the theoretical idea of CPTSD was originally suggested on the basis of female trauma victims' psychological difficulties (Herman, 1992). Future research should therefore explore cross-cultural characteristics as well as male populations in more depth, and the unique differences between men's and women's CPTSD symptoms.

This study has a number of limitations. The methodology is crosssectional; therefore, caution must be exercised in assuming causal explanations of the correlates associated with the CPTSD factors. In addition, neither the LEC-5 nor the CTS explicitly inquire about history of sexual abuse, thus we cannot know the contribution that this type of experience might have made to either symptom cluster in this population. The scales also do not include how frequent (i.e., one event or several events) or prolonged any of the traumatic experiences were. More specific measurements in future studies might enable more accurate results regarding the role of chronic trauma as compared to a one-time event, as a predictor of these diagnoses. Also, the childhood physical neglect construct in this study had a low level of reliability, perhaps due to the conceptual differences between the two scale items: the first referred more to the lack of physical needs being met, and the second to caregiver absence, which may be an indicator more of emotional than physical neglect. Therefore, further research should look at the roles of other types of childhood neglect in predicting CPTSD.

In Israel, there are many and varied cultural groups; the fact that we did not look at all of them in this study might have impacted the constructed model and the reliability of the Hebrew ITQ version. It is therefore essential to validate the ICD-11 diagnoses of both PTSD and CPTSD among additional cultural groups in Israel. Additionally, if social desirability was a goal for this mandated population (Chan, 2011), they might have reported less suffering than they actually experienced; low endorsement of symptoms related to their distress, such as the disturbed relationships cluster and the re-experiencing cluster, may have impacted the low CPTSD diagnosis rates.

This study's results clearly point to the need for further testing of the ICD-11 CPTSD ITQ in larger sections of the Israeli population, particularly among PTSD patients. To conclude, this study strengthens the validity of the ICD-11 PTSD and CPTSD diagnoses and is based on a unique population in a cultural context that has not previously been studied. It supports the necessity of the WHO diagnosis for use by clinicians and researchers who hope to better understand the distress experienced by clients exposed to traumatic events. It could also lead to a better assessment of a previously unstudied clinical population. The 
ongoing support of CPTSD construct validity should encourage clinicians to screen for DSO symptomatology, and emphasizes the need for treatment interventions that are specifically tailored to address these symptoms (Cloitre, 2015).

Funding for this research was provided in part by the Israel Ministry of Social Affairs and Social Services. The views expressed in this manuscript do not necessarily reflect the views of the funding agencies.

\section{References}

Ankri, Y. L., Bachar, E., \& Shalev, A. Y. (2010). Reactions to terror attacks in ultraOrthodox Jews: The cost of maintaining strict identity. Psychiatry, 73(2), 190-197.

Asparouhov, T., \& Muthén, B. (2010). Weighted least squares estimation with missing data. MplusTechnical Appendix [Retrieved from http://www.statmodel.com/download/ GstrucMissingRevision.pdf at 7 7.16].

Blake, D. D., Weathers, F. W., Nagy, L. M., Kaloupek, D. G., Klauminzer, G., Charney, D. S., \& Keane, T. M. (1990). A clinician rating scale for assessing current and lifetime PTSD: The CAPS-1. Behavior Therapy, 13, 187-188.

Brewin, C. R., Cloitre, M., Hyland, P., Shevlin, M., Maercker, A., Bryant, R. A., \& Reed, G. M. (2017). A review of current evidence regarding the ICD-11 proposals for diagnosing PTSD and complex PTSD. Clinical Psychology Review, 58, 1-15. http://dx.doi. org/10.1016/j.cpr.2017.09.001.

Briere, J. (2002). Treating adult survivors of severe childhood. The APSAC handbook on child maltreatment 175 .

Brown, T. A. (2006). Confirmatory factor analysis for applied research. New York: The Guilford Press.

Chan, K. L. (2011). Gender differences in self-reports of intimate partner violence: A review. Aggression and Violent Behavior, 16(2), 167-175.

Cloitre, M., Garvert, D. W., Brewin, C. R., Bryant, R. A., \& Maercker, A. (2013). Evidence for proposed ICD-11 PTSD and complex PTSD: A latent profile analysis. European Journal of Psychotraumatology, 4. http://dx.doi.org/10.3402/ejpt.v4i0.20706.

Cloitre, M., Roberts, N. P., Bisson, J. I., \& Brewin, C. R. (2015). The ICD-11 trauma questionnaire. Self-Report community version 1.5 [Unpublished measure].

Cloitre, M. (2015). The "one size fits all" approach to trauma treatment: Should we be satisfied? European Journal of Psychotraumatology, 6, 27344. http://dx.doi.org/10. 3402/ejpt.v6.27344.

Delsol, C., \& Margolin, G. (2004). The role of family-of-origin violence in men's marital violence perpetration. Clinical Psychology Review, 24(1), 99-122.

Derogatis, L. R., \& Melisaratos, N. (1983). The brief symptom inventory: An introductory report. Psychological Medicine, 13(3), 595-605.

Dokkedahl, S., Oboke, H., Ovuga, E., \& Elklit, A. (2015). ICD-11 trauma questionnaires for PTSD and complex PTSD: Validation among civilians and former abducted children in northern Uganda. Journal of Psychiatry, 18(335), 1-9. http://dx.doi.org/10.4172/ 23785756.1000335 .

Eisinga, R., Grotenhuis, M. T., \& Pelzer, B. (2012). The reliability of a two-item scale: Pearson Cronbach, or Spearman-Brown? International Journal of Public Health, 58(4), 637-642. http://dx.doi.org/10.1007/s00038-012-0416-3.

Elklit, A., Hyland, P., \& Shevlin, M. (2014). Evidence of symptom profiles consistent with posttraumatic stress disorder and complex posttraumatic stress disorder in different trauma samples. European Journal of Psychotraumatology, 5. http://dx.doi.org/10. 3402/ejpt.v5.24221.

Flora, D., \& Curran, P. J. (2004). An empirical evaluation of alternative methods of estimation for confirmatory factor analysis with ordinal data. Psychology Method, 9(4), $466-491$.

Ford, J. D., \& Courtois, C. A. (2014). Complex PTSD, affect dysregulation, and borderline personality disorder. Borderline Personality Disorder and Emotion Dysregulation, 1(1), 9-17.

Gilbar, O., \& Ben-Zur, H. (2002). Adult Israeli community norms for the brief symptom inventory (BSI). International Journal of Stress Management, 9(1), 1-10.

Gray, M. J., Litz, B. T., Hsu, J. L., \& Lombardo, T. W. (2004). Psychometric properties of the life events checklist. Assessment, 11(4), 330-341.

Hasherut Lerevahat Haprat VeHamishpaha (2016). The service for the welfare of the individual and the family. sicum peilut venetunim lishnat. 2015 Summery of activities and data of 2015. Israel: Social Welfare Ministry.

Herman, J. L. (1992). Trauma and recovery: The aftermath of violence from domestic violence to political terrorism. New York, NY: Guilford Press.

Hoyt, T., Wray, A. M., Wiggins, K. T., Gerstle, M., \& Maclean, P. C. (2012). Personality profiles of intimate partner violence offenders with and without PTSD. Journal of Offender Rehabilitation, 51(4), 239-256.

Hu, L., \& Bentler, P. M. (1999). Cutoff criteria for fit indexes in covariance structure analysis: Conventional criteria versus new alternatives. Structural Equation Modeling, 6(1), 1-55.

Hyland, P., Murphy, J., Shevlin, M., Vallières, F., McElroy, E., Elklit, A., \& Cloitre, M. (2017). Variation in post-traumatic response: The role of trauma type in predicting ICD-11 PTSD and CPTSD symptoms. Social Psychiatry and Psychiatric Epidemiology, 6, $1-10$.

Hyland, P., Shevlin, M., Brewin, C. R., Cloitre, M., Downes, A. J., Jumbe, S., \& Roberts, N. P. (2017). Validation of post-traumatic stress disorder (PTSD) and complex PTSD using the International Trauma Questionnaire. Acta Psychiatrica Scandinavica, 136(3), 313-322.

Hyland, P., Shevlin, M., Elklit, A., Murphy, J., Vallières, F., Garvert, D. W., \& Cloitre, M. (2016). An assessment of the construct validity of the ICD-11 proposal for complex posttraumatic stress disorder. Psychological Trauma: Theory Research Practice and
Policy, 9(1), 1-9.

Hyland, P., Shevlin, M., McNally, S., Murphy, J., Hansen, M., \& Elklit, A. (2016). Exploring differences between the ICD-11 and DSM-5 models of PTSD: Does it matter which model is used? Journal of Anxiety Disorders, 37, 48-53.

Karatzias, T., Shevlin, M., Fyvie, C., Hyland, P., Efthymiadou, E., Wilson, D., \& Cloitre, M. (2016). An initial psychometric assessment of an ICD-11 based measure of PTSD and complex PTSD (ICD-TQ): Evidence of construct validity. Journal of Anxiety Disorders, $44,73-79$.

Karatzias, T., Shevlin, M., Fyvie, C., Hyland, P., Efthymiadou, E., Wilson, D., \& Cloitre, M. (2017). Evidence of distinct profiles of post traumatic stress disorder (PTSD) and complex post traumatic stress disorder (CPTSD) based on the new ICD-11 trauma questionnaire (ICD-TQ). Journal of Affective Disorders, 207, 181-187.

Kline, R. B. (2011). Principles and practice of structural equation modeling (3rd ed.). New York: Guilford.

Knefel, M., \& Lueger-Schuster, B. (2013). An evaluation of ICD-11 PTSD and complex PTSD criteria in a sample of adult survivors of childhood institutional abuse. European Journal of Psychotraumatology, 4. http://dx.doi.org/10.3402/ejpt.v4i0.22608.

Knefel, M., Garvert, D. W., Cloitre, M., \& Lueger-Schuster, B. (2015). Update to an evaluation of ICD-11 PTSD and complex PTSD criteria in a sample of adult survivors of childhood institutional abuse by Knefel \& Lueger-Schuster (2013): A latent profile analysis. European Journal of Psychotraumatology, 6, 25290.

Krammer, S., Kleim, B., Simmen-Janevska, K., \& Maercker, A. (2015). Childhood trauma and complex posttraumatic stress disorder symptoms in older adults: A study of direct effects and social-interpersonal factors as potential mediators. Journal of Trauma \& Dissociation, 17(5), 593-607.

Lee, R. D., Walters, M. L., Hall, J. E., \& Basile, K. C. (2013). Behavioral and attitudinal factors differentiating male intimate partner violence perpetrators with and without a history of childhood family violence. Journal of Family Violence, 28(1), 85-94.

Maercker, A., Brewin, C. R., Bryant, R. A., Cloitre, M., Reed, G. M., Van Ommeren, M., \& Rousseau, C. (2013). Proposals for mental disorders specifically associated with stress in the International Classification of Diseases-11. The Lancet, 381(9878), 1683-1685.

Maguire, E., Macdonald, A., Krill, S., Holowka, D. W., Marx, B. P., Woodward, H., \& Taft, C. T. (2015). Examining trauma and posttraumatic stress disorder symptoms in courtmandated intimate partner violence perpetrators. Psychological Trauma: Theory Research Practice and Policy, 7(5), 473-478.

Murphy, S., Elklit, A., Dokkedahl, S., \& Shevlin, M. (2016). Testing the validity of the proposed ICD-11 PTSD and complex PTSD criteria using a sample from Northern Uganda. European Journal of Psychotraumatology, 7, 1-10. http://dx.doi.org/10.3402/ ejpt.v7.32678.

Muthén, L. K., \& Muthén, B. O. (2013). Mplus user's guide (7th ed.). Los Angeles, CA: Muthén \& Muthén.

Nickerson, A., Cloitre, M., Bryant, R. A., Schnyder, U., Morina, N., \& Schick, M. (2016) The factor structure of complex posttraumatic stress disorder in traumatized refugees. European Journal of Psychotraumatology, 8(1), 1-4.

Perkonigg, A., Höfler, M., Cloitre, M., Wittchen, H. U., Trautmann, S., \& Maercker, A. (2015). Evidence for two different ICD-11 posttraumatic stress disorders in a community sample of adolescents and young adults. European Archives of Psychiatry and Clinical Neuroscience, 266(4), 317-328.

Raykov, T. (1997). Estimation of composite reliability for congeneric measures. Applied Psychological Measurement, 21(2), 173-184.

Shevlin, M., Hyland, P., Karatzias, T., Fyvie, C., Roberts, N., Bisson, J. I., \& Cloitre, M. (2017). Alternative models of disorders of traumatic stress based on the new ICD-11 proposals. Acta Psychiatrica Scandinavica, 135(5), 419-428.

Silove, D., Tay, A. K., Kareth, M., \& Rees, S. (2017). The relationship of complex Post Traumatic Stress Disorder and Post-Traumatic Stress Disorder in a culturally distinct, conflict-affected population: A study among West Papuan refugees displaced to Papua New Guinea. Frontiers in Psychiatry, 8, 73 [10.3389/fpsyt.2017.00073 two prospective longitudinal studies. doi:10.1037/0002-9432.77.2.182].

Solomon, Z., \& Horesh, D. (2007). Changes in diagnostic criteria for PTSD: Implications from two prospective longitudinal studies. American Journal of Orthopsychiatry, 77(2), 182-188. http://dx.doi.org/10.1037/0002-9432.77.2.182.

Straus, M. A., Hamby, S. L., Boney-McCoy, S., \& Sugarman, D. B. (1996). The revised Conflict Tactics Scales (CTS2) development and preliminary psychometric data. Journal of Family Issues, 17(3), 283-316.

Taft, C. T., Schumm, J. A., Marshall, A. D., Panuzio, J., \& Holtzworth-Munroe, A. (2008). Family-of-origin maltreatment, posttraumatic stress disorder symptoms, social information processing deficits, and relationship abuse perpetration. Journal of Abnormal Psychology, 117(3), 637-646.

Taft, C. T., Murphy, C. M., \& Creech, S. K. (2016). Trauma-informed treatment and prevention of intimate partner violence. Washington, DC: American Psychological Association.

Tay, A. K., Rees, S., Chen, J., Kareth, M., \& Silove, D. (2015). The structure of posttraumatic stress disorder and complex post-traumatic stress disorder amongst West Papuan refugees. BMC Psychiatry, 15, 111-128. http://dx.doi.org/10.1186/s12888015-0480-3.

Van der Kolk, B. A. (1987). The psychological consequences of overwhelming life experiences. In B. A. Van der Kolk (Ed.). Psychological trauma (pp. 1-30). Washington: American Psychiatric Association.

Weathers, F. W., Blake, D. D., Schnurr, P. P., Kaloupek, D. G., Marx, B. P., \& Keane, T. M. (2013). The life events checklist for DSM-5 (LEC-5). [Retrieved from the National Center for PTSD at www.ptsd.va.gov.].

Wolf, E. J., Miller, M. W., Kilpatrick, D., Resnick, H. S., Badour, C. L., Marx, B. P., \& Friedman, M. J. (2014). ICD-11 complex PTSD in US national and veteran samples: prevalence and structural associations with PTSD. Clinical Psychological Science, 3(2), 215-229. 